\title{
Waterproof Liners for Hip Spica Casts Reduce Unplanned Healthcare Encounters
}

Elinor K. Berger, DNP, APRN, CPNP1,2; Ashley N. Startzman, DO' ; Hilda H. Kriel, $M D^{1}$

${ }^{1}$ Baylor Scott and White, Roney Bone and Joint Institute, Department of Orthopedic Surgery, Temple, TX; ${ }^{2}$ College of Nursing and Health Innovation, University of Texas Arlington, Arlington, TX

Correspondence to: Elinor K. Berger, DNP, APRN, CPNP; Department of Orthopedic Surgery, Baylor Scott and White, Roney Bone and Joint Institute, 2401 South 31st Street, Temple, TX 76508, E-mail: elinor.berger@bswhealth.org

Received: December 29, 2021; Accepted: January 17, 2022; Published: February 1, 2022

DOI: 10.55275/JPOSNA-2022-0013

\begin{abstract}
:
This quality improvement project evaluated implementation of the current American Academy of Orthopaedic Surgeons (2020) hip spica cast liner guidelines to reduce excess healthcare encounters associated with hip spica cast complications. The primary objective of this project was to identify if the use of a waterproof hip spica cast liner will reduce unplanned healthcare encounters related to hip spica cast complications.

A retrospective chart review was used to measure and compare the incidence of excess healthcare encounters associated with cotton and waterproof hip spica liners. Retrospective review of the electronic health record (EHR) identified pediatric patients between the ages of six months and six years of age who required a hip spica cast for the treatment of a diaphyseal femur fracture from January 1, 2020, through April 30, 2021. Unplanned healthcare encounters during the 90-day period beginning with hip spica cast application and abnormal skin assessment at cast removal was quantified using EHR review of hip spica casts that utilized cotton or waterproof liners. Chi-squared test showed statistically significant reduction in unplanned healthcare encounters and cast complications associated with waterproof hip spica cast liners. Utilization of waterproof hip spica cast liners decreased unplanned healthcare encounters by $100 \%(n=28)$ and significantly reduced skin complications associated with hip spica cast treatment.

Casting is one of the most common interventions utilized in pediatric orthopaedics. ${ }^{1}$ A common complication associated with cast treatment is the inability to dry the extremity. ${ }^{2}$ Cotton liners, traditionally used in cast applications, often trap moisture and chemical irritants such as urine, stool, and sweat resulting in adverse skin reactions, unplanned emergency room visits, nursing visits, cast adjustments, readmissions, and return to the operating room for cast change under anesthesia. ${ }^{3}$
\end{abstract}


Complications associated with hip spica casts result in unplanned healthcare encounters in excess of standard of care. ${ }^{4}$ Prior to 2020 , cotton liners were routinely used for hip spica casts at the project institution. All pediatric orthopaedic providers at the project institution utilized the same standardized protocol for diaphyseal femur fractures treated with a hip spica cast. Standard management for diaphyseal femur fractures at the project institution included four encounters: cast application, 1-week alignment check, cast removal at 4-6 weeks, and physical exam with radiographic follow up at 10-12 weeks (Table 1).

A feasibility study was performed at the project institution using a preliminary retrospective review of the electronic health record (EHR) over a 12-month period. The feasibility study identified 14 hip spica casts placed on pediatric patients $1-6$ years of age. Of the 14 hip spica casts identified, 12 exceeded the number of encounters associated with the institution's standard hip spica fracture management, 10 had abnormal skin complications in excess of expected cast irritation, four patients returned to the emergency department for cast concerns, and one patient returned to the operating room due to the presence of maggots in the cast. Unplanned healthcare encounters are used as an indicator of cast complications, patient experience, and cost based on the assumption that skin complications and complaints associated with a hip spica cast will result in additional healthcare utilization. ${ }^{3,4}$

\section{Literature Review}

PubMed was used to perform a comprehensive search to identify all English-language articles related to waterproof cast liners and hip spica casts. An initial search for hip spica cast yielded 254 results. Adding the terms waterproof and skin eliminated all search results. To appropriately narrow the search, the terms hip spica cast and skin resulted in 26 articles of which five applied to the identified clinical problem. Further search for waterproof cast liner resulted in an additional eight articles. A research request was placed for a librarian-assisted search that yielded 11 articles. Titles and abstracts were reviewed for relevance to the current project question and duplications within searches were identified. Articles were excluded if the focus was on indications of hip spica casts, if they lacked an association with skin assessment, or if waterproof liners were not evaluated. Eleven articles were selected based on the inclusion criteria of hip spica casts, waterproof liners, and skin evaluation.

Hip spica casts are standard treatment for multiple pediatric orthopaedic conditions that require immobilization of the femur and pelvis. ${ }^{5}$ In 2009 , the American Academy of Orthopaedic Surgeons

Table 1. Project Institution Protocol for the Management of Diaphyseal Femur Fractures using a Hip Spica Cast

\begin{tabular}{|l|l|l|l|l|l|}
\hline $\begin{array}{l}\text { Scheduled } \\
\text { Encounter }\end{array}$ & $\begin{array}{l}\text { Time from Cast } \\
\text { Application (weeks) }\end{array}$ & $\begin{array}{l}\text { Planned } \\
\text { Intervention }\end{array}$ & Normal Skin Assessment & Provider & Location \\
\hline 1 & 0 & $\begin{array}{l}\text { Spica Cast } \\
\text { Application }\end{array}$ & Baseline skin condition & MD & $\begin{array}{l}\text { Children's } \\
\text { Hospital OR }\end{array}$ \\
\hline 2 & 1 & $\begin{array}{l}\text { Alignment } \\
\text { check }\end{array}$ & Intact at cast edges & MD or NP & Pedi Ortho Clinic \\
\hline 3 & $4-6$ & Cast Removal & $\begin{array}{l}\text { Irritations consistent with casting. } \\
\text { No significant skin breakdown }\end{array}$ & MD or NP & Pedi Ortho Clinic \\
\hline 4 & $10-12$ & $\begin{array}{l}\text { Final } \\
\text { radiograph }\end{array}$ & Warm, dry, and intact & MD or NP & Pedi Ortho Clinic \\
\hline
\end{tabular}

Note. Adapted from the project institution pediatric orthopaedic MD/NP supervision and delegation protocol (C. Souder, personal communication, August 2016) 
(AAOS) created guidelines that recommended the use of waterproof liners during the application of pediatric hip spica casts. Although the guideline is based on limited evidence, the AAOS continues to support the recommendation since periodic review and subsequent studies have not yielded sufficient contrary evidence to change the guideline. ${ }^{6}$ To ensure an evidence-based intervention for the proposed clinical question, a review of the literature and current AAOS guidelines was necessary. Incorporation of published literature, clinical expertise, and patient experience is the cornerstone of evidence-based practice. ${ }^{7}$

\section{Population}

The utilization of hip spica casts define the age-group population affected by the clinical problem. Used to treat various orthopaedic conditions, hip spica casts are primarily used on children 6 months through 6 years of age. ${ }^{8}$ The population affected by this clinical problem is therefore limited to this age group. Review of the literature specifically related to hip spica casts includes only a pediatric population. However, cast complications are not unique to pediatric hip spica casts. To expand the evaluation of cast complications, articles related to cast types associated with similar complications were included. Additionally, review of the literature included studies that evaluated skin integrity, patient satisfaction, and cost. Since all age groups are included in other forms of cast immobilization, studies involving non-hip spica cast types contain adult age group data.

The paucity of pediatric data frequently limits application of pediatric drugs and devices; thus, the Food and Drug Administration leverages relevant adult data in cases where pediatric data may be limited. ${ }^{9}$ Results from the Dubowitz and Miller ${ }^{10}$ case report and Guillen et al. ${ }^{2}$ waterproof cast material study were based on adult study participants and must be extrapolated for pediatric patients. Of the 11 primary articles reviewed, Guillen et al. ${ }^{2}$ included adult age group data, and Dubowitz and Miller ${ }^{10}$ provided a case report of one adult's experience with a waterproof cast liner. Guillen et al. ${ }^{2}$ used a randomized crossover design to compare patient preference of waterproof versus cotton cast liners. Although adults were included in the study sample, pediatric patients accounted for $90 \%(\mathrm{n}=18)$ of study participants. Guillen et al. ${ }^{2}$ did not have sufficient participation to derive a statistical difference in adult and child participant preference. Using an adult case report, Dubowitz and Miller ${ }^{10}$ validated evidence that a Gortex liner successfully mitigated complications associated with moisture and improved patient satisfaction. Cast complications related to moisture are not unique to pediatric patients, therefore, the inclusion of an adult case report was deemed appropriate to broaden the scope of the literature review.

Indications for hip spica casts are not evaluated in this literature review. The AAOS (2020) continues to provide guidelines for the use of age-appropriate hip spica casts for various orthopaedic conditions. According to the AAOS guidelines (2020), the use of a waterproof liner does not affect the previously established indications for the use of a hip spica cast. This literature review did not identify a primary study directly comparing the efficacy of waterproof lined versus cotton lined hip spica casts to maintain reduction of femoral shaft fractures. However, DiFazio et al. ${ }^{4}$ did evaluate skin complications associated with hip spica casting for the treatment of pediatric femur fractures. DiFazio et al. ${ }^{4}$ noted all fractures united appropriately regardless of liner type, but this was not measured or discussed in greater detail. Wolf and James ${ }^{11}$ evaluated children treated with hip spica casts for developmental dysplasia of the hip. The authors did not find any differences in treatment efficacy when they compared hip spica casts lined with a Gortex Pantaloon liner versus a traditional cotton stockinette liner. ${ }^{11}$ Tisherman et al. ${ }^{12}$ acknowledge the ability of waterproof liners to reduce the need for unplanned cast changes in their systematic review of hip spica casts for the treatment of diaphyseal femur fractures. However, they did not review any prior studies evaluating reduction effectiveness of hip spica casts with waterproof or cotton liners. Robert et al. ${ }^{13}$ demonstrated equivalent effectiveness of cotton versus waterproof cast liners in the ability to maintain acceptable reduction of 
previously displaced pediatric distal forearm fractures. The most recent review article generalizes the results of Robert et al. ${ }^{13}$ and recognizes hip spica casts that utilize waterproof liners have fewer skin complications and did not review evidence suggestive of decreased efficacy with the treatment of femur fractures. ${ }^{3}$

\section{Intervention}

The most common complication associated with hip spica casts is skin breakdown associated with moisture in the cast. ${ }^{12}$ DiFazio et al. ${ }^{4}$ identified soiling as the cause of $81 \%(n=63)$ of skin complications associated with hip spica casts. Even with the most diligent care, hip spica casts are extremely difficult to keep dry. The traditional cotton stockinet liner can absorb, transmit, and retain moisture. $^{4}$

The use of a waterproof liner for hip spica casts facilitates the evaporation of moisture and promotes skin integrity during cast treatment. ${ }^{3}$ Since the introduction of the Gortex cast liner in the early 1990s, several waterproof cast liner products are available for use. ${ }^{14}$ Stevenson et al. ${ }^{14}$ compared pediatric short arm casts with Wet or Dry ${ }^{\circledR}$, or Delta Dry ${ }^{\circledR}$ waterproof cast liners in terms of patient satisfaction and comfort, cast technician satisfaction during application and removal, as well as drying time. Stevenson et al. ${ }^{15}$ found qualitative results for patient preferences were similar for both products. The cast technician preferred Delta Dry ${ }^{\circledR}$, but only a single technician was surveyed. ${ }^{15}$ Both Stevenson et al. ${ }^{15}$ and Guillen et al. ${ }^{2}$ used a randomized prospective design to compare cast liners in short arm casts. The crossover design utilized by Guillen et al. ${ }^{2}$ strengthened the results that demonstrate similar fracture outcomes regardless of liner type. Although multiple studies have compared waterproof liners to standard cotton liners, no other literature was found to directly compare multiple waterproof liners used for hip spica casts. Prior results suggest waterproof liners are a safe alternative intervention to reduce cast complications associated with moisture without compromising orthopaedic outcomes. ${ }^{2,4,11,13,16}$ Skin complications, patient satisfaction, and cost are the predominating themes throughout this literature review of waterproof cast liners and hip spica casts.

\section{Skin Complications}

Hip spica cast skin complications can result in local and systemic infections, hospital readmission, and costly return trips to the operating room for cast change. . $^{41,12,17}$ The significance of this complication is highlighted repeatedly throughout the literature and is the main rational for the need for an effective alternative to the cotton lined hip spica cast. ${ }^{411,12,15-18}$ Although the study was of limited quality, Kruse et al. ${ }^{16}$ was one of the first to assess skin integrity after casts lined with Gortex fabric compared to a cotton liner. In addition to hip spica casts, Kruse et al. ${ }^{16}$ included clubfoot casts to compare the Gortex liner on one leg and the cotton liner on the contralateral side. This method allowed for the patients to act as their own control. Although the results were limited by sample size and lack of detailed outcome measures, the observation of improved hygiene spurred the further investigation by Wolff and James. ${ }^{11}$

A landmark study by Wolff and James ${ }^{11}$ documented $31 \%$ (11 of 36) of cotton lined hip spica casts had significant skin complications. DiFazio et al. ${ }^{4}$ substantiated these findings with a much larger sample size. DiFazio et al. ${ }^{4}$ reported $28 \%(n=77)$ of cotton lined hip spica casts experienced skin complications. Similarly, Guillen et al. ${ }^{2}$ found cotton lined upper extremity casts also had statistically more skin irritation than the Gortex lined group. DiFazio et al. ${ }^{18}$ later performed a large, interrupted time series study to identify the incidence of cast-related skin complications. DiFazio et al. ${ }^{18}$ evaluated almost 17,000 various cast types and found the incidence of skin complications to be 13.6 per 1000 casts. Although they did determine the most common types of skin complications over all types of casts, DiFazio et al. ${ }^{18}$ did not evaluate hip spica casts or moisture-related complications specifically. Hip spica casts are associated with moisture-related skin complications, but they are not used as frequently as other types of cast immobilization. DiFazio et al. ${ }^{18}$ established a baseline rate of cast-related skin complications, but when they used descriptive 
statistics and Pareto charts to analyze the most frequent skin complications, the more common single leg lower extremity cast was identified for their improvement intervention.

Even though hip spica casts are not used as frequently as other cast types, skin complications are common, and complications can be severe. Tisherman et al. ${ }^{12}$ evaluated hip spica cast studies for the treatment of diaphyseal femur fractures. Systematic review noted skin complications as the most common complication associated with hip spica cast treatment. Metaanalyses of the decade of spica cast studies noted skin complications were twice as frequent as fracture complications associated with hip spica casts. ${ }^{12}$

The case report by Chan et al. ${ }^{17}$ highlights the potentially severe health risks associated with wet hip spica casts. Moisture in this child's hip spica was significant enough to grow fungi within the cast overlying a postoperative wound. ${ }^{17}$ This case report emphasized the significant risk of systemic fungal infection when moisture is retained in a hip spica cast utilized for postoperative pelvis and hip immobilization in children.

The comparative study by Stevenson et al. ${ }^{15}$ demonstrated the ability of waterproof cast liners to minimize skin complications associated with cast moisture. The two brands of waterproof liners studied were effective and comparable regarding skin integrity under the high demands of hygiene and recreational water activities in pediatric patients with odds ratio of 0.21 at $95 \%$ confidence interval and $p$-value $<0.001 .^{15}$ Guillen et al. ${ }^{2}$ documented statistically significant improvement in skin condition of waterproof lined casts compared to cotton lined casts with a two-tailed $p$ value of .038. These results further support the ability of waterproof cast liners to minimize skin complications associated with casting.

\section{Satisfaction}

Since the Institute of Medicine (IOM) introduced the idea of patient-centered healthcare in 2001, patient satisfaction is increasingly used as a metric to measure healthcare quality. ${ }^{19}$ The importance of the patient experience is supported by evidence that relates high patient satisfaction with improved compliance and clinical outcomes. ${ }^{19}$ Increased utilization of unplanned healthcare encounters is associated with parental concerns, perception of urgency, and the need for reassurance. ${ }^{20}$

Patient satisfaction is the cornerstone of several studies evaluating waterproof cast liners. Shirley et al. ${ }^{3}$ recognized the burden to families, patients, and societal issues surrounding cast wear. Specifically, hip spica casts were most difficult for families to perform adequate hygiene, associated with unplanned clinic and emergency room visits, and required multiple missed work and school days. ${ }^{3}$ Systematic review of the literature by Tisherman et al. ${ }^{12}$ also recognized parental complaints surrounding hip spica casts. Parents reported difficulty with patient hygiene, cast soilage, and increased weight of the cast. Their review of the literature reported waterproof liners resulted in fewer cast changes due to soilage, compared to cotton liners, implying its association with improved hygiene. ${ }^{12}$ Five studies reviewed incorporated parental or patient experiences and perceptions into their design. ${ }^{2,10,13,15,16}$ Although these studies utilized the subjective report of patient experience as part of the study, there has not been a standardized, validated questionnaire. Prior to Guillen et al., ${ }^{2}$ previous studies did not have a comparison group when measuring patient-rated scores. Guillen et al. ${ }^{2}$ attempted to control for personal variance by utilizing a prospective randomized crossover design. Their results supported Kruse et al. ${ }^{16}$ and Dubowitz and Miller's ${ }^{10}$ suggestion that patients had improved satisfaction with waterproof lined casts and preferred them over cotton lined casts $75 \%(n=20)$ of the time. Stevenson et al. ${ }^{15}$ compared two brands of waterproof cast liners and found that patients enjoyed water activities and improved hygiene but did not have a significant preference between brands.

\section{Cost}

With innovation and technology comes rising cost. Waterproof cast liners are more expensive than their 
cotton counterparts. ${ }^{10}$ AquaCast LLC $^{14}$ reports an increased cost of approximately $\$ 100$ per waterproof hip spica cast liner. Stevenson et al. ${ }^{15}$ acknowledge cost difference between different brands of waterproof liners. To justify the increased cost of waterproof liners, the literature reviewed compares these liners to the cost of poor outcomes related to wet cotton cast liners and the associated healthcare utilization.

An unplanned cast change increases the total cost of any type of cast treatment. The cost of an unplanned hip spica cast change in the operating room is greater than a cast change that can occur in the clinic setting. DiFazio et al. ${ }^{4}$ calculated an average cost of $\$ 12,719$ for each cast change due to skin complications of hip spica cast treatment. This increased from Wolff and James ${ }^{11}$ calculation of $\$ 10,500$ over 15 years earlier. Healthcare costs for skin complications associated with hip spica casts increases over time. Both Wolff and James ${ }^{11}$ and DiFazio et al. ${ }^{4}$ calculated and compared total treatment cost of hip spica casts lined with a waterproof liner with hip spica casts lined with a traditional cotton liner. In both studies, the waterproof hip spica liner was found to be significantly cost effective compared to cotton lined hip spica casts. ${ }^{4,11}$

Additional charges associated with wet casts include emergency department visits, clinic and nurse visits, and the time away from work, school, and travel associated with these unplanned visits. ${ }^{4}$ Guillen et al. ${ }^{2}$ demonstrated decreased skin irritation with the use of waterproof cast liners. The crossover nature limited the time in the casts for each participant since they were switched midway through immobilization treatment. This prevented Guillen et al. ${ }^{2}$ from evaluating unplanned cast changes. The results from Guillen et al. ${ }^{2}$ supports the rational that fewer skin complications would result in fewer unplanned healthcare encounters during hip spica cast treatment. Systematic review by Tisherman et al. ${ }^{12}$ further supports the prior claim that waterproof cast liners result in fewer hip spica cast changes due to soilage. The most recent review of waterproof cast liners reiterates that although initially more expensive than cotton cast liners, the cost savings related to decreased unplanned cast changes and other unplanned healthcare encounters offsets this increased cost. ${ }^{3}$

\section{Framework}

Rosswurm and Larrabee's ${ }^{21}$ model for evidence-based practice change provides a logical framework to guide implementation of the proposed hip spica cast project. The first step in this model allows for a comparison of internal data related to skin integrity and hip spica cast liners with external data from the literature. This author's pediatric orthopaedic department observed increased skin integrity compromise with the use of traditional cotton liners for hip spica cast application. Step two links the use of cotton cast liners with poor skin integrity. Synthesized evidence supports the proposed waterproof liner intervention with reduced adverse skin reactions, unplanned visits, and unplanned cast changes. Step three synthesizes the current AAOS guidelines and recent primary research into the proposed hip spica liner practice change. The project design exchanges the cotton cast liner for the waterproof liner during the application of hip spica casts. Step four uses available evidence to define the practice change and needed resources to ensure optimal opportunity to improve the objective outcomes. The fifth step evaluates both process and clinical outcomes to best determine if waterproof liners improve patient outcomes through decreased unplanned healthcare encounters. Project results determine if the change should be adopted, adapted, or rejected. The decision to integrate the waterproof cast liner as part of the department's hip spica cast protocol will be based on objective results of this hip spica cast project. The Rosswurm and Larrabee ${ }^{21}$ model provides a framework to translate a good idea to practice specific implementation.

\section{Specific Aims}

This quality improvement project addresses the problem of increased healthcare utilization associated with hip spica casts. Does the use of a waterproof liner for pediatric patients who require a hip spica cast result in decreased cast-related unplanned healthcare 
encounters within the 90-day period after spica cast application?

\section{Project Objective}

The primary objective of this project is to identify if the use of a waterproof hip spica cast liner will reduce unplanned healthcare encounters during the 90-day period after hip spica application. The secondary objective will determine if the incidence of skin breakdown at cast removal is reduced with the use of a waterproof hip spica cast liner.

\section{Methods}

\section{Project Design}

A strength, weaknesses, opportunities, and threats (SWOT) analysis was performed prior to project design (Figure 1). Pediatric orthopaedic providers and clinic administration recognized the need to improve patient outcomes associated with hip spica casts. Clinic administrators acquired the preferred waterproof hip spica cast liner material as requested by the surgeons. Delta Dry ${ }^{\circledR}$ Pantaloon waterproof hip spica liners were made available to surgical staff, and education was provided to increase awareness of product availability. The BSN medical Delta Dry ${ }^{\circledR}$ Pantaloon ${ }^{22}$ patient education brochure was provided to parents (see Appendix A). Discharge instructions related to home care of the waterproof hip spica cast liner were updated.

Current AAOS (2020) hip spica cast liner guidelines were implemented to reduce excess healthcare encounters associated with hip spica cast complications. A retrospective chart review was used to measure and compare the incidence of excess healthcare encounters associated with cotton and waterproof hip spica liners over a 24-month period.

\section{Population and Setting}

Between April 2019 and April 2021, retrospective convenience samples were taken of pediatric patients between the ages of 6 months and 6 years who required a hip spica cast to treat a diaphyseal femur fracture. Each patient presented to a single not-for-profit children's hospital from which the hospital's EHR was used to collect the data. All patients sampled were cared for by the sole pediatric orthopaedic practice within the Children's Hospital system. Hip spica casts were applied to patients under general anesthesia in the operating room. A board certified orthopaedic surgeon, with assistance from an orthopaedic resident or certified cast technician, performed each application. Post-hospital

\begin{tabular}{|c|c|}
\hline Strengths & Weaknesses \\
\hline $\begin{array}{l}\text { - Orthopaedic providers eager to make a change in } \\
\text { hip spica cast protocol } \\
\text { - Surgeons had prior experience with waterproof } \\
\text { hip spica liners } \\
\text { - Administration open to trying a new product } \\
\text { - No budget restraints associated with new product }\end{array}$ & $\begin{array}{l}\text { - New product requires supply chain approval } \\
\text { - On-call surgeons unaware of product availability } \\
\text { or unfamiliar with product application } \\
\text { - Waterproof liner requires different postoperative/ } \\
\text { discharge instructions }\end{array}$ \\
\hline Opportunities & Threats \\
\hline $\begin{array}{l}\text { - Improved patient/caregiver experience } \\
\text { - Reduced skin complications } \\
\text { - Product available and easily acquired } \\
\text { - Minimal change to current hip spica cast protocol } \\
\text { - Cost savings associated with reduced utilization } \\
\text { and decreased complications }\end{array}$ & $\begin{array}{l}\text { - Increased initial cost of waterproof hip spica liner } \\
\text { - Cast care performed at home (outside the control } \\
\text { of the healthcare environment) } \\
\text { - Incorporating additional product training } \\
\text { (surgeon application and nurse discharge } \\
\text { education) with demands of clinical care }\end{array}$ \\
\hline
\end{tabular}

Figure 1. SWOT analysis table hip spica QI project. 
discharge hip spica cast management was provided by the same pediatric orthopaedic team. Excluded from this project were patients with hip spica casts applied to manage orthopaedic conditions not associated with diaphyseal femur fracture, patients who required open surgical management, patients with pre-existing skin injury to the casted area, and casts applied at an outside institution.

\section{Measurement Methods}

The project author identified and evaluated the incidence of unplanned healthcare encounters that exceeded institutional treatment protocol for hip spica casts. Unplanned healthcare encounters that exceed the standard of care were measured from review of EHR documentation using the data collection spreadsheet Unplanned Healthcare Encounter(s) Associated with Hip Spica Casts (Table 1). For the purposes of this project, an unplanned healthcare encounter is defined as any patient-initiated interaction with the healthcare system related to the hip spica cast that exceeds the project institution's standard planned encounters for hip spica cast fracture management (Table 2). During the 90-day period following hip spica cast application, any additional healthcare encounter beyond the standard was considered an unplanned, excess healthcare encounter.

The data collection spreadsheet (Table 2) was utilized to identify the absence or presence of abnormal skin assessment after cast removal. Additionally, Table 2 utilized the EHR data to count and categorize all unplanned healthcare encounters related to the hip spica cast, including emergency room visits, pediatric orthopaedic clinic visits, cast technician and nurse visits, telephone calls, patient portal messages, and return trips to the operating room. The chief complaint documented in the EHR categorized caregiver rationale for additional healthcare utilization and determined if the unplanned healthcare encounter was related to the hip spica cast. Nominal-level measurement supports the objectives for this project and is intended to document deviations from standard care.

\section{Data Collection and Implementation Plan}

In March 2020, waterproof liners for hip spica casts were made available to the cast cart in the children's operating room. E-mail communication was used to inform and remind orthopaedic trauma surgeons and residents associated with the Children's Hospital system of the availability, location, and application of the hip spica cast waterproof liners.

Email communication was also sent to surgeons, residents, and nurses at the children's hospital providing a commercially available patient education brochure. The institution's pediatric orthopaedic surgeons began using the waterproof hip spica liners in July 2020. If the appropriately sized waterproof liner was not available at the time of cast application, cotton liners and current cotton-lined hip spica patient education continued to be used during the project timeframe. Orthopaedic surgeons, residents, and nurses provided liner-specific hip spica cast care instructions during postoperative discussion and hospital discharge teaching.

The EHR used for data collection contains records of all healthcare encounters for the largest not-for-profit healthcare system in Texas, including encounters involving the emergency department, hospital admissions, operating room, outpatient clinic visits, urgent care, telephone calls, patient portal encounters, and virtual visits. ${ }^{23}$ Mitus 3.0 software was utilized in conjunction with an EHR report request to identify all electronic health records of hip spica cast applications by current procedural terminology codes 29325 and 29305 from April 2019 through April 2021. A feasibility study performed prior to the project determined the institution applies approximately one to two hip spica casts per month. A 24-month retrospective review was necessary to ensure the maximum number of waterproof lined hip spica casts were compared to a similar number of cotton-lined hip spica casts. The project author collected healthcare encounter data by utilizing a retrospective EHR review of pediatric patients treated for diaphyseal femur fractures with hip spica casts at the project institution. Each chart meeting inclusion criteria between 


\begin{tabular}{|c|c|c|c|c|c|c|c|c|c|c|c|c|}
\hline 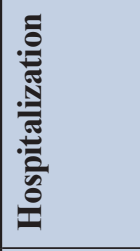 & & $\nabla$ & & & & & & & & & - & \\
\hline 高 & & - & & & & & & & & & & \\
\hline 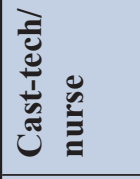 & & & & & & & & & & & & - \\
\hline 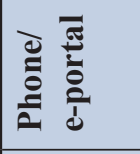 & & $m$ & & & & $\sim$ & & - & & - & - & - \\
\hline 苞 & & - & & & & & & & & & & \\
\hline 国 & & - & & & & & & & & - & - & \\
\hline 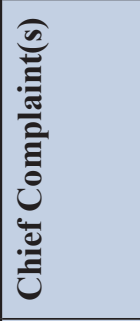 & $\stackrel{\pi}{\beth}$ & 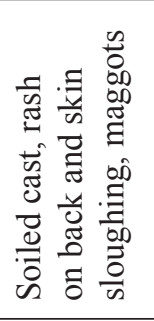 & 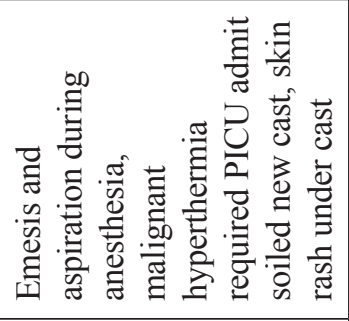 & $\stackrel{\pi}{a}$ & $\stackrel{\pi}{a}$ & 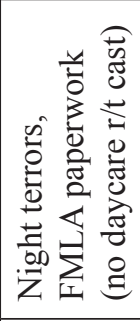 & $\stackrel{\pi}{\Xi}$ & 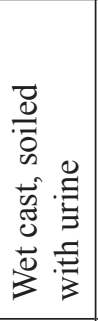 & $\stackrel{\pi}{a}$ & 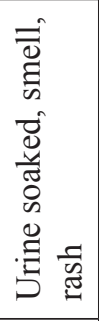 & 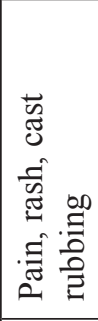 & 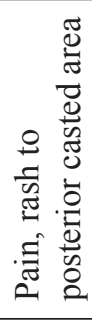 \\
\hline 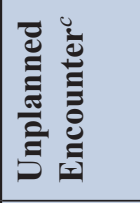 & 0 & $\stackrel{\circ}{-}$ & & 0 & 0 & $\sim$ & 0 & - & 0 & $\sim$ & $m$ & $\sim$ \\
\hline 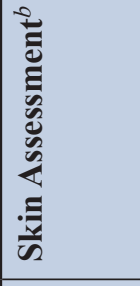 & 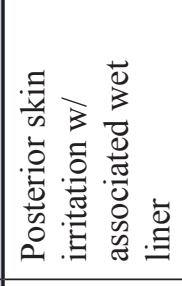 & 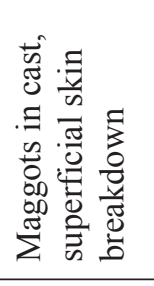 & 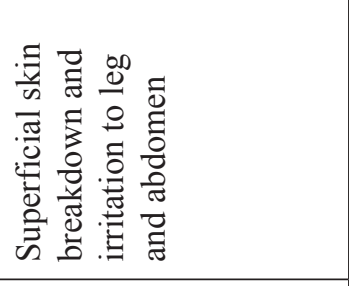 & 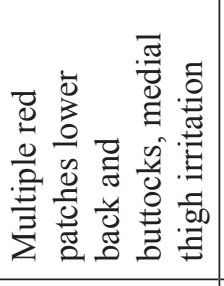 & 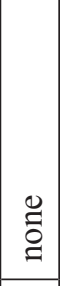 & 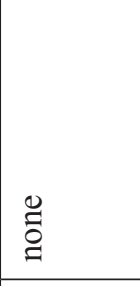 & $\underset{\Xi}{\Xi}$ & 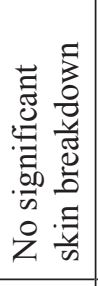 & $\underset{\Xi}{\Xi}$ & 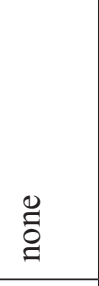 & 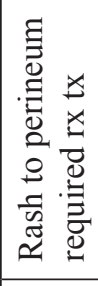 & 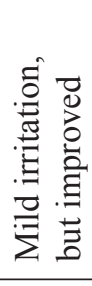 \\
\hline 离 & 0 & U & $U$ & $\cup$ & 3 & 0 & 3 & $U$ & 3 & $U$ & 0 & $U$ \\
\hline 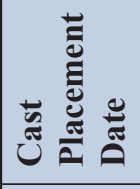 & 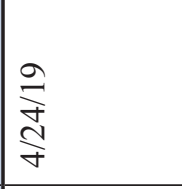 & $\underset{\substack{\text { I } \\
\text { I }}}{ }$ & 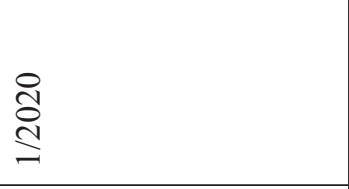 & 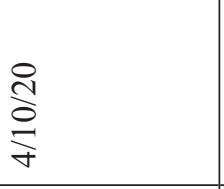 & 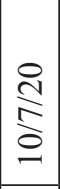 & \begin{tabular}{|l} 
N \\
N \\
d
\end{tabular} & 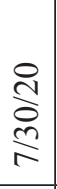 & 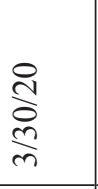 & 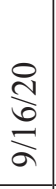 & 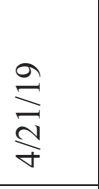 & 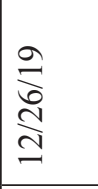 & 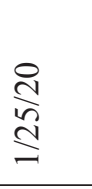 \\
\hline 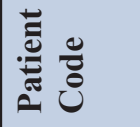 & - & $\sim$ & & & $\nabla$ & in & 0 & $r$ & $\infty$ & $a$ & $\stackrel{0}{0}$ & $=$ \\
\hline
\end{tabular}




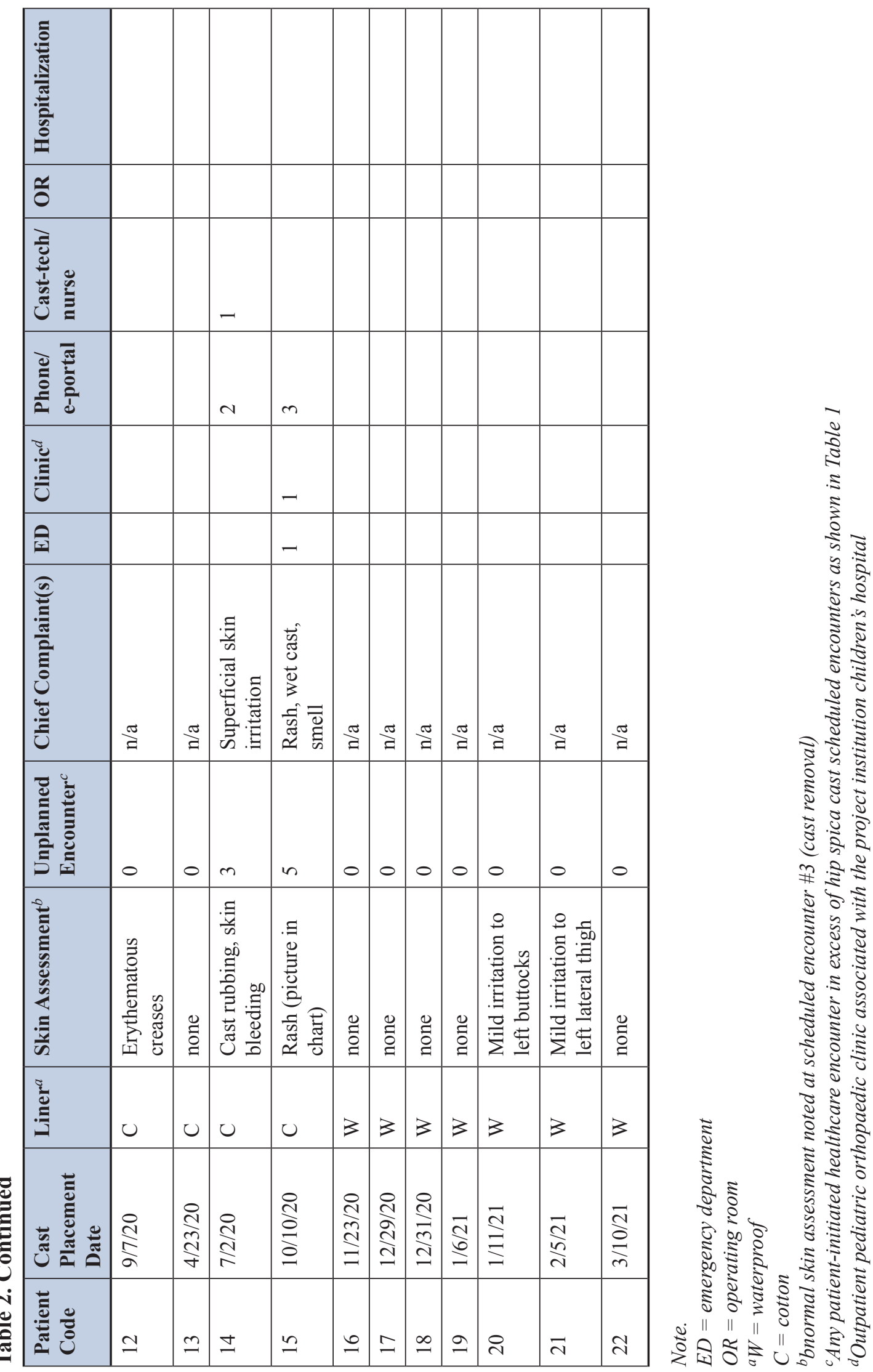


April 2019 and April 2020 was reviewed for the 90-day period following application of the hip spica cast. Data collected for the 90-day period beginning with hip spica cast application was organized on the spreadsheet Unplanned Healthcare Encounter(s) Associated with Hip Spica Casts. Liner type was identified by reviewing the EHR operative note documenting cast application. The number and type of unplanned healthcare encounters, chief complaint for each unplanned encounter, and skin condition upon cast removal was recorded in the spreadsheet (Table 2). Upon completion, collected data was divided into two cohorts: hip spica casts with cotton liners and hip spica casts with waterproof liners.

The 22 patient records that met inclusion criteria were used to describe each unplanned healthcare encounter. The EHR documentation from each unplanned encounter identified descriptive categories shown in Table 2. The EHR documentation was reviewed and deviations from the standard of care schedule and expected skin condition at hip spica cast removal (Table 1) were noted as abnormal. Unscheduled encounters and abnormal skin assessments represented unexpected healthcare utilization. The project scope did not stratify deviations from standard care. All data was de-identified and protected on an encrypted, password-protected hard drive, which remains with the project author. This project was fully supported as a quality improvement initiative by the project institution's pediatric orthopaedic department staff.

\section{Ethics}

The hip spica cast liner project utilized committee review to ensure an ethical project. The Graduate Nursing Review Committee, authorized by the University Internal Review Board (IRB), determined this to be a quality improvement or evidence-based implementation and approved the implementation of this project. The institution's nursing research committee reviewed and recommended the above project. This project was undertaken as a quality improvement initiative at Baylor Scott and White Roney Bone and Joint Institute; therefore, it was not formally supervised by their Institutional Review Board per their policies on quality improvement initiatives. Human subject training was completed prior to the implementation of the project. There were no conflicts of interest identified.

\section{Results}

Retrospective review of the EHR from April of 2019 through April of 2021 revealed 23 hip spica casts placed on 22 unique patients at the project institution. Of the 22 patients who met inclusion criteria, 12 used cotton stockinette liners while 10 utilized waterproof hip spica liners (Table 3).

Cotton liners were utilized for both hip spica casts on the patient who required cast replacement. All EHR encounters during the 90-day period after cast application were reviewed. The documented chief complaint for each encounter was used to determine if the encounter was related to the hip spica cast. Twenty-eight unplanned healthcare encounters related to the hip spica cast were identified. Of the 28 unplanned healthcare encounters, 20 different chief complaints were documented.

Recurrent chief complaint themes included rash, soiled and moisture, odor, pain, and other cast-related chief complaints unique to individual patients (Table 4). Individual patients' chief complaints included night terrors, anesthesia complications, maggots, and caregiver medical leave form request.

The 28 unplanned healthcare encounters consisted of four (14\%) emergency room visits, two (7\%) clinic visits with an orthopaedic provider, 14 (50\%) patient-initiated phone calls or patient portal communications, two (7\%) cast tech or nurse visits, five (17\%) inpatient hospitalization days,

Table 3. Liner Type Complications

\begin{tabular}{|l|l|l|}
\hline & Cotton & Waterproof \\
\hline Hip Spica Cast Patients & 12 & 10 \\
\hline Unplanned Encounters & 28 & 0 \\
\hline $\begin{array}{l}\text { Skin Complications at Cast } \\
\text { Removal }\end{array}$ & 8 & 2 \\
\hline
\end{tabular}


Table 4. Unplanned Healthcare Encounter Chief Complaint Themes

\begin{tabular}{|l|l|}
\hline Chief Complaint Theme & n (\%) \\
\hline Rash & $7(35)$ \\
\hline Soiled/Moisture & $5(25)$ \\
\hline Odor & $2(10)$ \\
\hline Pain & $2(10)$ \\
\hline Other $^{a}$ & $4(20)$ \\
\hline
\end{tabular}

Note. ${ }^{a}$ Chief complaints unique to an individual patient: night terrors, FMLA request, anesthesia complications, and maggots

and one $(3.6 \%)$ return trip to the operating room. Cotton lined hip spica casts were associated with 28 cast-related unplanned healthcare encounters while waterproof lined hip spica casts were associated with none (Figure 2).

The use of a waterproof liner reduced unplanned healthcare encounters by $100 \%(n=28)$.

A chi-square test of independence was performed to examine the relation between cast liner and unplanned healthcare encounters. A statistical difference in the number of patients that had unscheduled healthcare encounters was identified between the cotton and waterproof groups, $X^{2}(1, N=22)=10.48, \mathrm{p}=.001$ (Table 5).

All 28 unscheduled healthcare encounters occurred in the cotton lined group, with the most frequent contacts being related to rash $(35 \%)$, moisture $(25 \%)$, odor $(10 \%)$, and

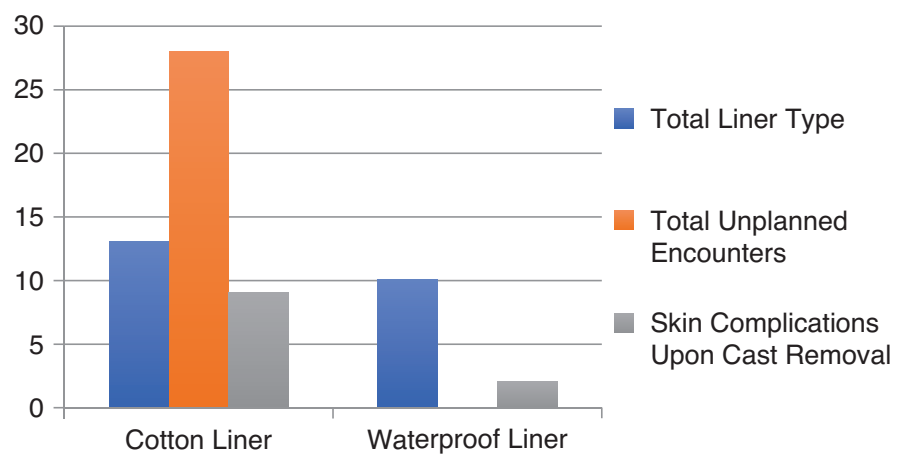

Figure 2. Unplanned encounters and skin complications by liner type.

pain (10\%). The remaining $20 \%$ of the chief complaints were unique to the presenting patient (Table 4). While most reasons for unplanned healthcare visits were relatively minor, at least one serious complication was noted. One child required a return trip to the operating room for cast change due to the presence of maggots in the severely soiled cast. In addition to skin breakdown, the subsequent anesthesia event resulted in significant complications including aspiration and malignant hyperthermia, which required pediatric intensive care admission.

In addition to healthcare utilization, provider assessment of skin status at cast removal was explored. Similar to the unplanned healthcare encounter findings, a statistically significant difference in skin breakdown at cast removal between the cotton and waterproof groups exists, $X^{2}$ $(1, N=22)=4.79, \mathrm{p}=.029$ (Table 6).

Significant skin breakdown was noted upon removal of nine cotton lined casts and two waterproof lined casts. Cotton liners were used for the child who required cast replacement and upon removal of each cast, skin breakdown was noted (Table 2). Although severity of skin breakdown was not measured in this project, significant breakdown requiring prescription treatment, such as bleeding lesions and rashes, were noted in the cotton group. The skin breakdown associated with two

Table 5. Unplanned Encounters by Liner Type

\begin{tabular}{|l|l|l|l|}
\hline Liner & No & Yes & Total \\
\hline Cotton & 4 & 8 & 12 \\
\hline Waterproof & 10 & 0 & 10 \\
\hline Total & 14 & 8 & 22 \\
\hline
\end{tabular}

Table 6. Skin Breakdown by Liner Type

\begin{tabular}{|l|l|l|l|}
\hline Liner & No & Yes & Total \\
\hline Cotton & 4 & 8 & 12 \\
\hline Waterproof & 8 & 2 & 10 \\
\hline Total & 12 & 10 & 22 \\
\hline
\end{tabular}


waterproof liners was documented as mild irritation. In addition to statistical significance, this author recognized the possibility that the use of a waterproof liner for hip spica casts may reduce rare but potentially serious untoward events.

\section{Discussion}

In summary, waterproof hip spica cast liners significantly reduce healthcare utilization by reducing unplanned encounters. Additionally, the use of waterproof hip spica liner helped maintain skin integrity. Although not directly measured, reduction of excess healthcare encounters implies reduced cost, equivalent efficacy related to fracture healing, and improved patient experience during hip spica cast treatment.

The associated benefits of waterproof hip spica liners were supported throughout the literature. Previous studies associated unplanned encounters with additional healthcare costs. Similar to this project's findings, DiFazio et al. ${ }^{4}$ and Wolff and James ${ }^{11}$ noted additional charges associated with wet casts include emergency department visits, clinic and nurse visits, and time away from work, school, and travel. The additional cost of each specific unplanned encounter was not measured but assumed to add to the overall cost of hip spica cast treatment. The unfortunate patient who experienced significant complications associated with a subsequent anesthesia event generated the most unscheduled encounters for a single patient. Project results suggest that this costly utilization could be avoided through the use of waterproof hip spica liners. The ability to reduce costs associated with both major and minor complications associated with cotton-lined hip spica casts more than offsets the initial cost of a waterproof hip spica liner.

Caregiver difficulty with patient hygiene, cast soilage, and the size and shape of the hip spica cast have been associated with unscheduled clinic and emergency room visits and multiple missed work and school days. ${ }^{3}$ Caregiver concerns associated with unscheduled encounters suggest healthcare utilization may be related to patient satisfaction. Although this project did not directly measure patient satisfaction, the $100 \%$ reduction in unscheduled healthcare encounters suggests that patients who utilized a waterproof hip spica liner had a better experience than those patients with cotton lined casts.

A systematic review noted skin complications as the most common complication associated with hip spica cast treatment. ${ }^{12}$ Consistent with previous studies by Stevenson et al. ${ }^{15}$ and Guillen et al., ${ }^{2}$ this project demonstrated waterproof liners reduce skin complications associated with hip spica cast treatment. This project did not assess fracture outcomes but decreased healthcare utilization in the waterproof liner group suggests equivocal fracture healing compared to the cotton lined group. This assumption is supported by Guillen et al. ${ }^{2}$ who demonstrated similar upper extremity fracture outcomes regardless of liner type.

The use of waterproof hip spica cast liners was found to be beneficial to the patient and the organization. The reduction in unplanned healthcare utilization and reduced complications associated with hip spica casts resulted in the decision to continue to utilize waterproof liners for all hip spica cast applications at the project institution.

\section{Limitations}

The retrospective chart review study design limits the generalizability of the results and could be associated with misclassification bias. Although this project demonstrated a $100 \%(n=28)$ reduction of unscheduled healthcare encounters, it is unlikely that a larger sample size would maintain complete elimination of all extra healthcare encounters. A larger sample size would allow for increased accuracy of true reduction rates. Additionally, the association with unscheduled healthcare encounters as they relate to cost, patient satisfaction, and treatment efficacy is based on prior literature but not directly measured in this project.

\section{Conclusion}

This quality improvement initiative has shown that the use of waterproof hip spica cast liners is associated with the reduction of unscheduled healthcare encounters 
and skin complications associated with hip spica cast treatment. The association with reduced major and minor complications support the cost effectiveness and sustainability of the waterproof hip spica liner product. The project findings support continued use of waterproof liners for hip spica cast application at the project institution. The project results raise the question if application of waterproof liners for other cast types would be beneficial at the project institution.

\section{Additional Links}

- Delta Dry ${ }^{\circledR}$ Pantaloon-Parent Information Film: https://youtu.be/19TLSCkUtvk ${ }^{22}$

- POSNAcademy video on application of waterproof single leg hip spica cast: https://www.posnacademy.org/media/Video+Abstract+8A+Fully+Wate rproof + One-legged + Spica + Cast + for + Femur + Fractu res/1_uedzjuw4/162962082 24

\section{Author's Note}

This QI project was performed at Baylor Scott and White Roney Bone and Joint Institute as a DNP project affiliated with the University of Texas Arlington College of Nursing and Health Innovation. SQUIRE 2.0 guidelines were used to prepare this quality improvement manuscript. ${ }^{25}$ No outside financial support was used.

\section{Acknowledgements}

The author would like to thank Cynthia Casey, DNP, RN, Faculty Project Adviser; Joy Don Baker, PhD, RN-BC, CNE, CNOR, NEA-BC, FAORN, FAAN, Writing Coach; and Jared F. Benge, PhD, ABPP, Statistician.

\section{References}

1. Wenger DR, Pring ME, Pennock AT, et al. Rang's Children's Fractures (4 ${ }^{\text {th }}$ ed.). Philadelphia, PA: Wolters Kluwer; 2018.

2. Guillen PR, Fuller CB, Riedel BB, et al. A prospective randomized crossover study on the comparison of cotton versus waterproof cast liners. Hand. 2016;11(1):50-53.

3. Shirley ED, Maguire KJ, Mantica AL, et al. Alternatives to traditional cast immobilization in pediatric patients. $J$ Am Acad Orthop Surg. 2020;1(28):e20-e27. doi: https://doi.org/10.5435/JAAOS-D-18-00152.

4. DiFazio R, Vessy J, Zurakowski D, et al. Incidence of skin complications and associated charges in children treated with hip spica casts for femur fractures. J Pediatr Orthop. 2011;31(1):17-22. doi: https://doi. org/10.1097/BPO.0b013e3182032075.
5. Bitar KM, Ferdhany ME, Ashraf EI, et al. Physical and clinical evaluation of hip spica cast applied with three-slab technique using fiberglass material. Malays Orthop J. 2016;10(3):17-20. doi: https://doi.org/10.5704/ MOJ.1611.008

6. American Academy of Orthopaedic Surgeons. Treatment of pediatric diaphyseal femur fractures evidence-based clinical practice guideline. 2020. Available at https://www.aaos.org/globalassets/quality-and-practiceresources/pdff/pdffcpg.pdf.

7. Fineout-Overholt E, Stillwell SB, Williamson KM, et al. Teaching evidence-based practice in academic settings. In: Melnyk B, FineoutOverholt E, eds. Evidence-Based Practice in Nursing and Healthcare. Philadelphia, PA: Wolters Kluwer; 2019:445-496.

8. Herring JA. Tachdjian's Pediatric Orthopaedics from the Texas Scottish Rite Hospital for Children (4 $4^{\text {th }}$ ed., Vol. 1). Saunders Elsevier; 2008.

9. U.S. Department of Health and Human Services, Food and Drug Administration, Center for Devices and Radiological Health, Center for Biologics Evaluation and Research. Leveraging existing clinical data for extrapolation to pediatric uses of medical devices (FDA Publication No. 1827), 2016. Retrieved from https://www.fda.gov/media/91889/download.

10. Dubowitz G, Miller DM. Cast adrift: Gortex cast liners allow greater patient activity. Wilderness Environ Med. 2003;14(3):167-168. doi: https:// doi.org/10.1580/1080-6032(2003)14.

11. Wolff CR, James P. The prevention of skin excoriation under children's hip spica casts using the Goretex pantaloons. J Pediatr Orthop. 1995;15(3):386-388. doi: https://doi.org/10.1097/01241398-199505000-0.

12. Tisherman RT, Hoellwarth JS, Mendelson SA. Systematic review of spica casting for the treatment of paediatric diaphyseal femur fractures. J Child Orthop. 2018;12(2):136-144. doi: https://doi. org/10.1302/1863-2548.12.170201.

13. Robert CE, Jiang JJ, Khoury JG. A prospective study on the effectiveness of cotton versus waterproof cast padding in maintaining the reduction of pediatric distal forearm fractures. J Pediatr Orthop. 2011;31(2):144-149. doi: https://doi.org/10.1097/BPO.0b013e318209d83a.

14. American Academy of Orthopaedic Surgeons. Treatment of pediatric diaphyseal femur AquaCast Liner LLC. AquaCast Hipster Pantaloon liners, 2017. Available at: https://aquacastliner.com/product-category/ hipster-protective-liners/.

15. Stevenson AW, Gahukamble AD, Antoniou G, et al. Waterproof cast liners in paediatric forearm fractures: A randomized trial. J Child Orthop. 2013;7(2):123-130. doi: https://doi.org/10.1007/s11832-012-0472-5.

16. Kruse RW, Fracchia M, Boos M, et al. Gortex fabric as a cast underliner in children. J Pediatr Orthop. 1991;11(6):786-787.

17. Chan Y, Selvaratnam V, Garg NA. A fungating spica. BMJ Case Reports. Online Jan 2015;1-3. doi: https://doi.org/10.1136/bcr-2014-206901.

18. DiFazio RL, Harris M, Feldman L, et al. Reducing the incidence of cast related skin complications in children treated with cast immobilization. J Pediatr Orthop. 2017;37(8):526-531. doi: https://doi.org/10.1097/ BPO.0000000000000713.

19. Smith MJ, Choma TJ. Patient satisfaction in musculoskeletal medicine. Curr Rev Musculoskelet Med. 2017;10(2):207-211. doi: https://doi. org/10.1007/s12178-017-9403-x.

20. Nicholson E, McDonnell T, De Brun A, et al. Factors that influence family and parental preferences and decision making for unscheduled paediatric healthcare: Systematic review. BMC Health Serv Res. 2020;20:663. https://doi.org/10.1186/s12913-020-05527-5.

21. Rosswurm MA, Larrabee, JH. A model to change evidence-based practice. J Nurs Scholarsh. 1999;31(4):317-322. doi: https://doi. org/10.1111/j.1547-5069.1999.tb00510.x.

22. BSN Medical. Delta-Dry ${ }^{\circledR}$ Pantaloon-Parent Information Film. February 1, 2017. Retrieved from https://youtu.be/19TLSCkUtvk.

23. Baylor Scott and White Health. About Baylor Scott and White Health: Our story. 2020. Available at https://www.bswhealth.com/about/pages/ default.aspx. 
24. Minkowitz B, Ristic JR, Mendelow E, et al. Video abstract 8: Fully waterproof one-legged spica cast for femur fractures. POSNAcademy Annual Meeting 2020-Video Abstracts.

25. Ogrinc G, Davies L, Goodman D, et al. SQUIRE 2.0 Standards for Quality Improvement Reporting Excellence: Revised publication guidelines from a detailed consensus process. BMJ Qual Saf. 2016;25(12):986-992. doi: https://doi.org/10.1136/bmjqs-2015-004411.

26. BSN Medical GmbH. Delta-Dry ${ }^{\mathbb{R}}$ : PANTALOON Improving Life During Hip Spica Cast Treatment, 2017. [Brochure] Essity. 


\section{Appendix A}

Delta-Dry ${ }^{\circledR}$ : PANTALOON Improving Life During Hip Spica Cast Treatment

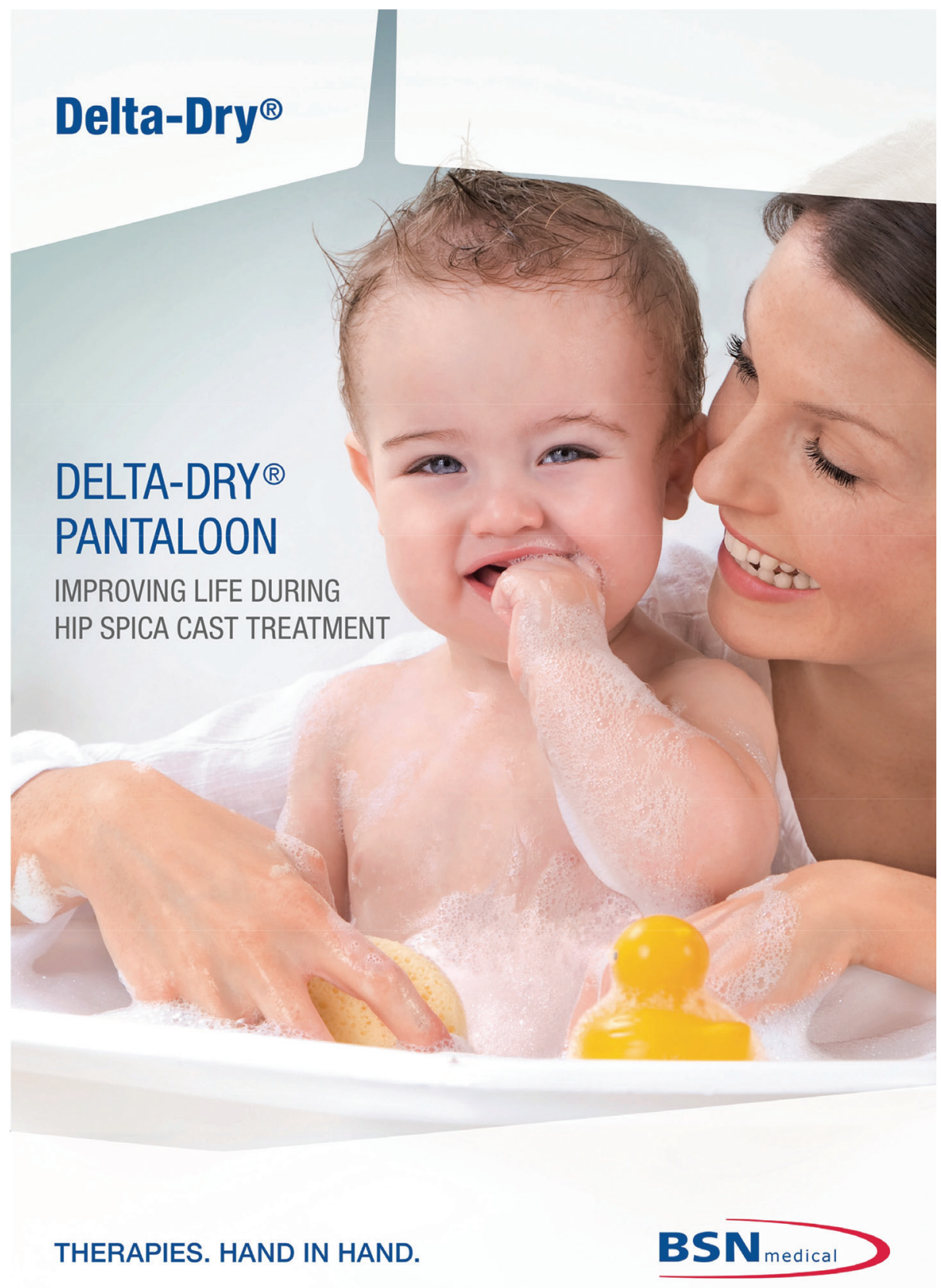




\section{DAILY ROUTINE WITH A HIP SPICA CAST}

MAINTAINING YOUR CHILD'S HYGIENE

\section{Dear Parents,}

BSN medical appreciates that any medical treatment is a challenging time for any parent and their child. You probably have a lot of questions on how to care for your child during a hip spica cast treatment. Your health care professional will provide you with all necessary information and will answer all your questions. But as an additional aid, we'd like to provide you with some information on how to look after your child during this time.

Keeping baby's skin and the cast clean during hip spica cast treatment has been quite a difficult task until now. As your child has been fitted with a special hip spica cast made with the water-resistant undercast material Delta-Dry ${ }^{\circ}$ Pantaloon, residual smell through leftover soiling hygiene routines belong to the past.

Pre-shaped especially for hip spica cast treatments, Delta-Dry ${ }^{\circledast}$ Pantaloon can get fully wet and will be dry within $\mathbf{9 0}$ minutes. This means you can flush out or clean away any soiling and even bathe your child. You will reduce the risk of skin complication while maintaining your child's daily hygiene routine.

It's our commitment to ease your way through hip spica cast treatment. We hope you will find this guide useful and wish you and your child a speedy and successful treatmen. Your BSN medical

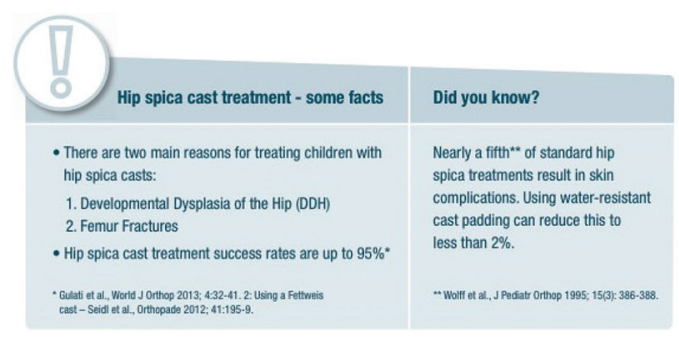

\section{DESIGNED WITH YOUR BABY IN MIND}

BENEFITS OF DELTA-DRY® PANTALOON

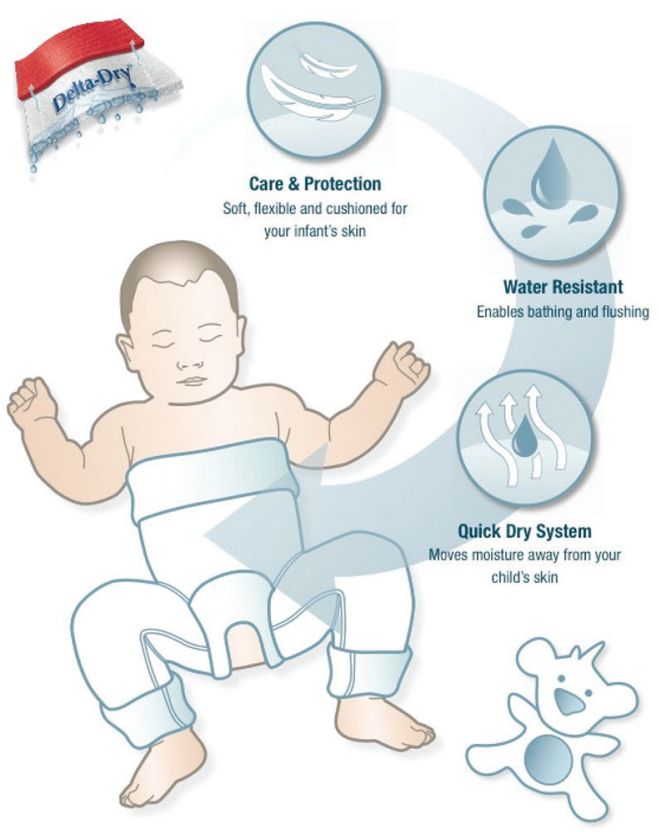

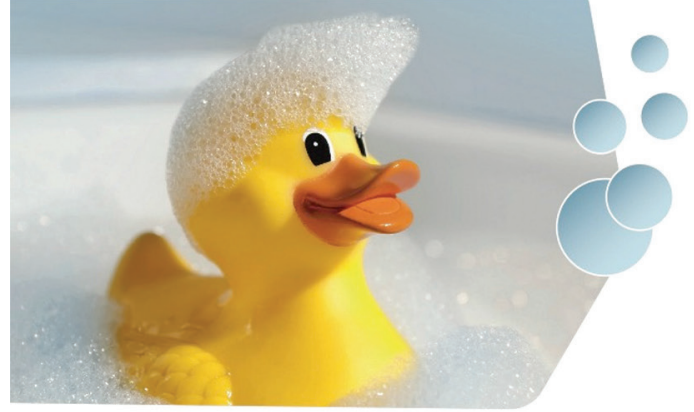

\section{BATHING FUN}

HYGIENE MADE EASY

Thanks to Delta-Dry Pantaloon, maintaining your baby's hygiene is a little easier than it would be with a traditional hip spica cast.

Little accidents no longer mean more cast changes than necessary. Flushing the cas through, or a bath, will help to keep your baby and the hip spica cast clean

For best results, please follow these rules

- To keep the skin as healthy as possible, flush the cast through with clear. lukewarm water every two to five days.

- For bathing choose, for example, a bath tub or a sink.

- Use mild soap and afterwards always rinse with clear, lukewarm water.

- If the cast gets soiled by any urine or excrement, wash the affected areas immediately.

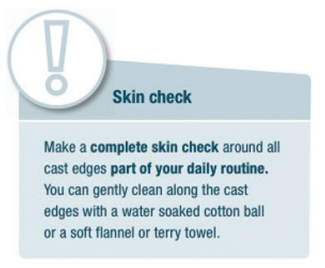

\section{QUICK DRY SYSTEM}

DRYING AFTER BATHING OR FLUSHING

The Pantaloon's unique material allows skin to breathe while carrying out any moisture when wet. After a splash in the bath tub, there are some things to remember to ensure

the cast fully dries:

- Let all water drain out of the leg openings of the cast.

- Change the position of your child every 10 minutes during drying to avoid so called pooling ie, the accumblation of water in one place. This will help to speed up the drying process.

- Any remaining moisture will evaporate due to your child's body heat.

- Ensure to allow enough time for the cast within ry. The cast should be dry

- During drying, keep your child in a warm room, so it does not get cold - but do ot covertitwih clothes, blankets from drying properly.

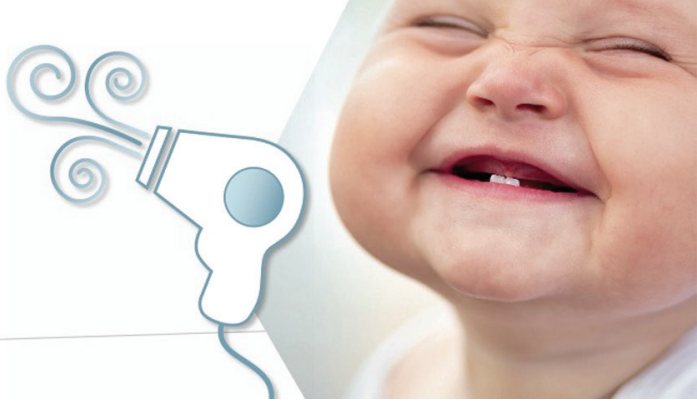


ADVANCED DIAPERING

TWO ARE BETTER THAN ONE

There are several ways of diapering your little one during hip spica cast treatment. Here is one proposal for which you will need two different sizes of diapers your regular size, and one larger).

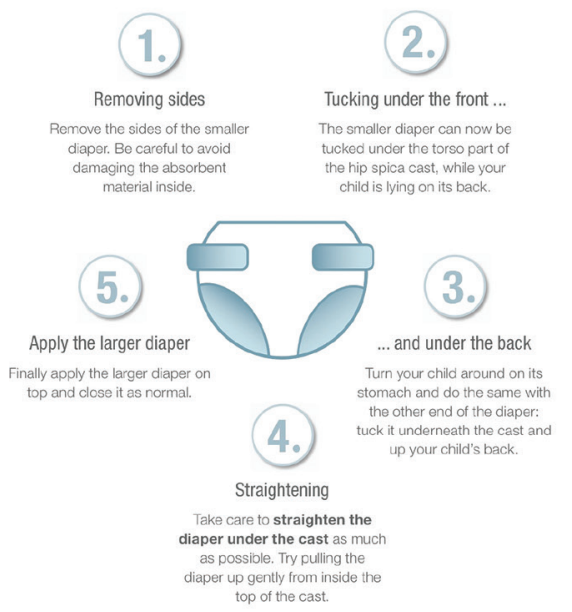

5.)

$$
\begin{aligned}
& \text { stomach and do tho same with } \\
& \text { the other end of the diaper: } \\
& \text { tuck it tunderneaeth the cast and } \\
& \text { up your child's back. }
\end{aligned}
$$

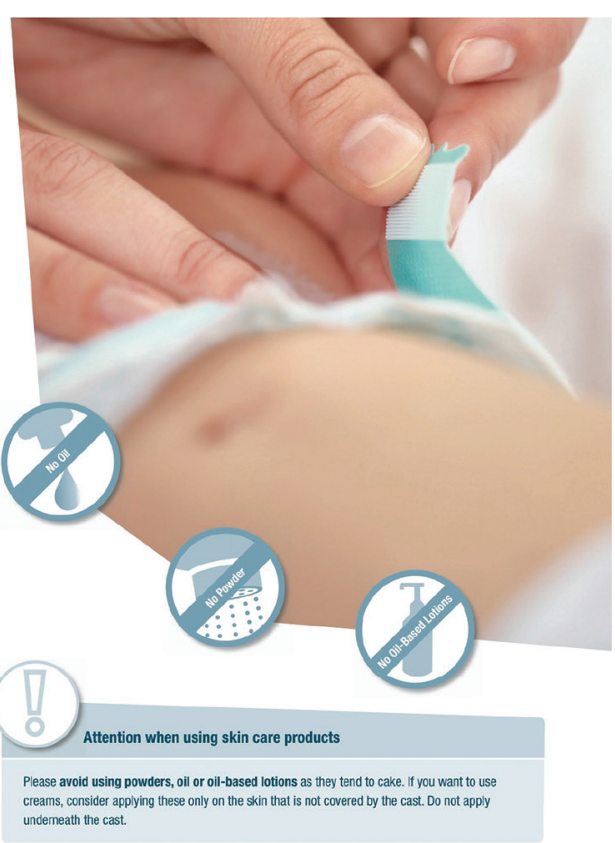

BSN

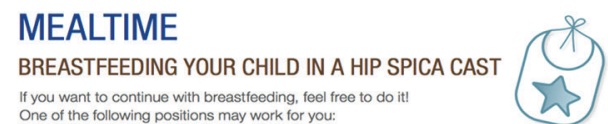

One of the following positions may work for you:

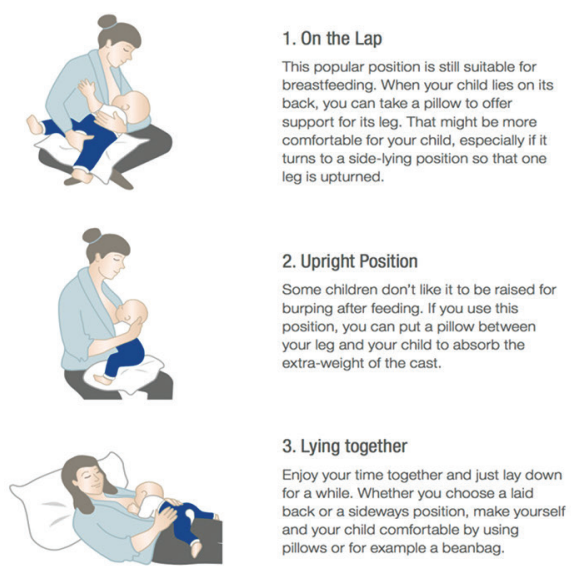

IN THE CAR

PREPARATIONS FOR TRAVEL BETWEEN HOME AND HOSPITAL A suitable child seat is essential - with or without a hip spica cast. For the treatment duration it might be necessary to adapt the seating to your child's needs. The seat
should have a flat base and low sides. Please also check that any car seat meets the should have a flat base and low sides. Please also check that any car seat meets the age, weight and height of your child.

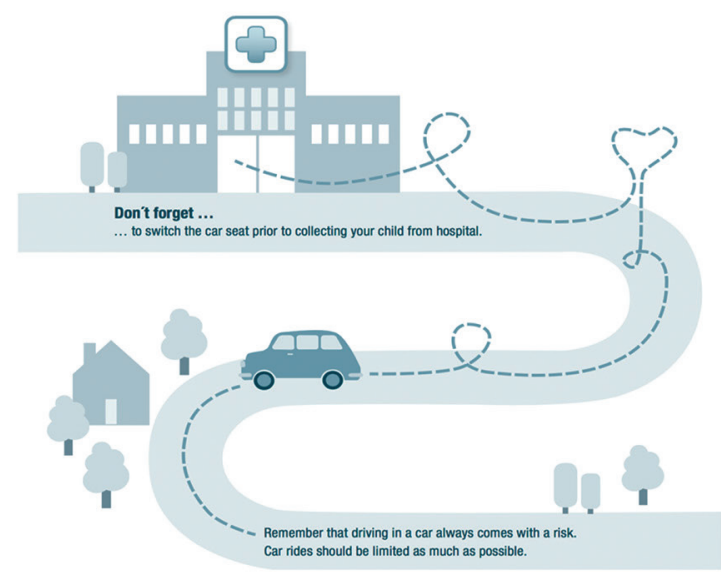




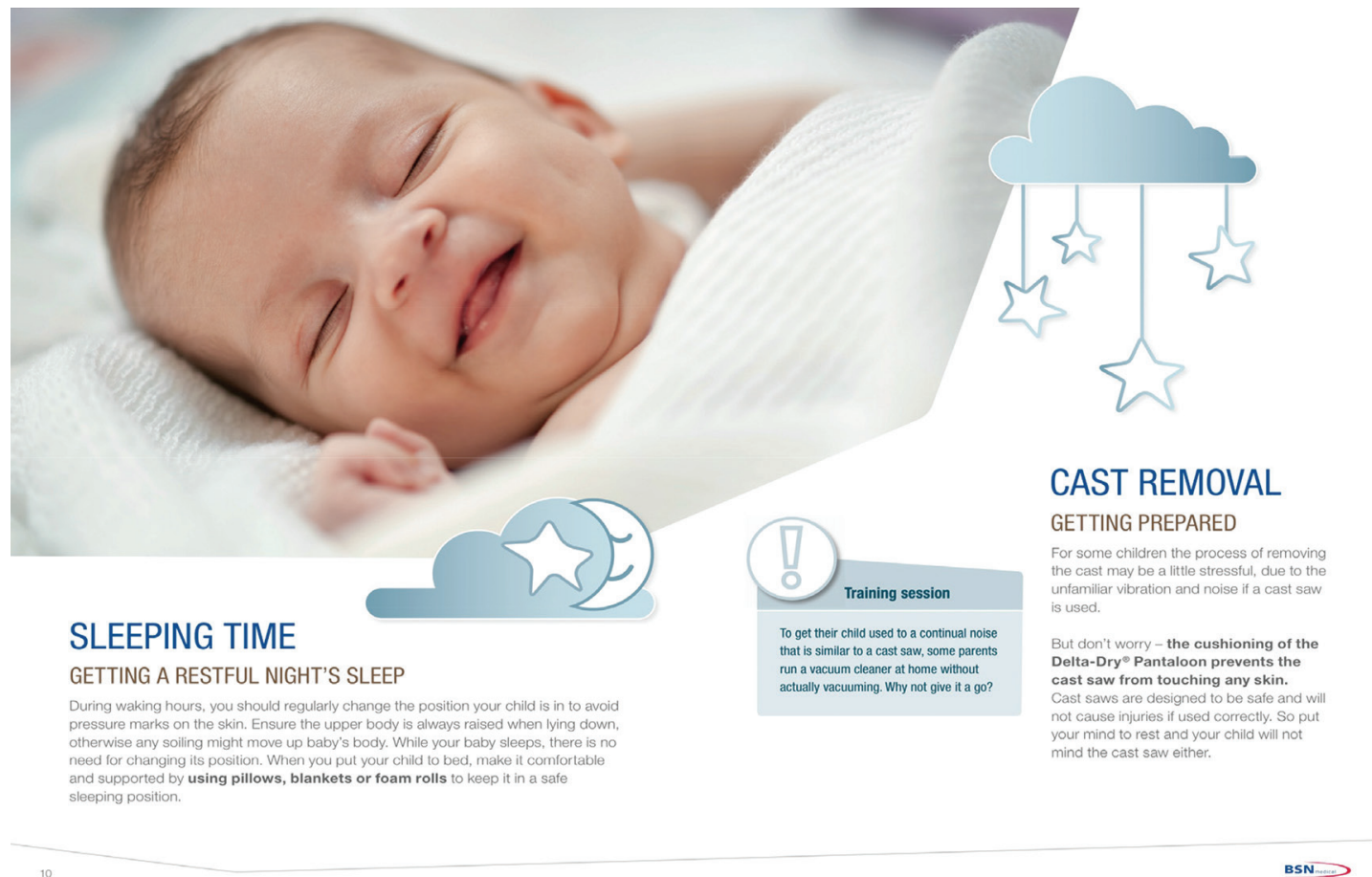

\section{FIRST AID}

WHAT TO DO IF YOUR BABY FEELS ITCHING

Try out to re-position your child. If that doesn't work. you can also try to help by washing your child or

blowing with a fan or hair dryer on a cold setting in the

direction of the cast - please ensure, that your child

enjoys it.

Do not attempt to scratch your child's skin

under the cast. Also, do not insert any items.

This poses a huge risk for skin complications.
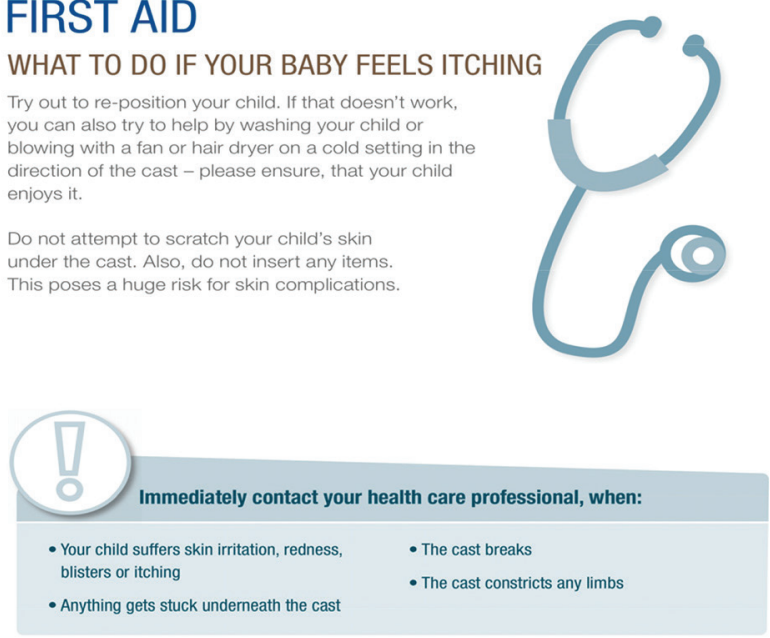

BSN medical GmbH

Quickbornstraße 24
20253 Hamburg

www.bsnmedical. com
Tel. $+4940 / 4909-4909$
Fax $+49800 / 850-1021$

BSN

Note. Printed brochure from BSN Medical GmbH ${ }^{26}$ Essity. Reprinted with permission. Permission granted for use in manuscript from Essity North America (A. Bartus, personal e-mail communication, August 11, 2021). 